\title{
Diagnóstico patológico y molecular de un caso de tuberculosis laríngea primaria en un médico
}

\author{
Leonardo F. Jurado ${ }^{1,2}$, Diana M. Palacios ${ }^{1,3}$, Johanna Álvarez ${ }^{1}$, Margarita Baldión", Guillermo Campos ${ }^{4}$ \\ 1 Departamento de Patología y Laboratorios, Fundación Santa Fe de Bogotá, Bogotá, D.C., Colombia \\ 2 Grupo de Micobacterias, Departamento de Microbiología, Facultad de Medicina, Universidad Nacional de Colombia \\ Colombia, Bogotá, D.C., Colombia \\ ${ }^{3}$ Departamento de Patología, Facultad de Medicina, Universidad Nacional de Colombia, Bogotá, D.C., Colombia \\ ${ }^{4}$ Instituto de Laringología, Asociación Médica de los Andes, Fundación Santa Fe de Bogotá, Bogotá, D.C., Colombia
}

La tuberculosis laríngea primaria es una enfermedad rara con una amplia gama de manifestaciones clínicas. Se reporta el caso de un médico otorrinolaringólogo con diabetes de tipo II y con diagnóstico de tuberculosis laríngea primaria. El diagnóstico de cáncer de cuerda vocal se había considerado en otra institución, y al ingreso el paciente sólo presentaba disfonía de seis meses de evolución. En el estudio histopatológico se encontraron granulomas, células gigantes multinucleadas, fibrosis y necrosis, y en la tinción de Ziehl-Neelsen, bacilos ácido-alcohol resistentes. El cultivo del tejido de cuerda vocal para micobacterias y la PCR-IS6110 fueron positivos. La cepa de Mycobacterium tuberculosis demostró sensibilidad a todos los medicamentos de primera línea. Se inició tratamiento según la estrategia DOTS (Directly Observed Treatment, Short-course). Se discute la presentación clínica, los diagnósticos diferenciales, el abordaje diagnóstico y los factores de riesgo asociados y se hace una breve revisión de la literatura.

Palabras clave: Mycobacterium tuberculosis, reacción en cadena de la polimerasa, tuberculosis laríngea, granuloma, disfonía.

doi: http://dx.doi.org/10.7705/biomedica.v34i1.1624

\section{Pathological and molecular diagnosis in a case of primary laryngeal tuberculosis in a physician}

Primary laryngeal tuberculosis is a rare disease with a wide variety of clinical manifestations. We report the case of an ear-nose and throat diabetic physician with primary laryngeal tuberculosis. The diagnosis of laryngeal cancer had been considered before in another institution, and at the patient's admission, he only presented dysphonia with a six-month evolution. The vocal cord histological examination showed granulomas, giant cells, fibrosis and necrosis, and the Ziehl-Neelsen staining showed acid-alcohol resistant bacilli. The culture from vocal cord tissue was positive for mycobacteria, and IS6110-PCR was positive too. The Mycobacterium tuberculosis strain was sensitive to first line drugs. Treatment using directly observed therapy short-course (DOTS) was initiated. Clinical presentation, differential diagnosis, diagnostic workup, associated risk factors and a brief literature revision are discussed in this article.

Key words: Mycobacterium tuberculosis, polymerase chain reaction, laryngeal tuberculosis, granuloma, dysphonia.

doi: http://dx.doi.org/10.7705/biomedica.v34i1.1624

\section{Correspondencia:}

Leonardo F. Jurado, Grupo de Micobacterias, Departamento de Microbiología, Facultad de Medicina, Universidad Nacional de Colombia, Carrera 30 № 45-03, edificio 471, piso 3, Bogotá, D.C., Colombia

Teléfono: (312) 8737570

Ifjuradoz@unal.edu.co

Recibido: 17/04/13; aceptado:05/09/13
La tuberculosis es una enfermedad muy antigua, pero no es una enfermedad del pasado (1). Diversos estudios plantean que Mycobacterium tuberculosis ha estado presente sobre la faz de la tierra alrededor de 15.000 años, afectando al Homo sapiens por milenios (2). En las décadas de 1960 y 1970, la tuberculosis había desaparecido de las agendas mundiales de salud pública y los países desarrollados hablaban de la eliminación

\section{Contribución de los autores:}

Guillermo Campos: realización de los procedimientos quirúrgicos, revisión del manuscrito.

Diana Palacios, Johanna Álvarez y Margarita Baldión: realización del estudio histopatológico, revisión y comentario del manuscrito. Leonardo Jurado: revisión de la historia clínica y la literatura científica pertinente, interpretación de los análisis moleculares microbiológicos y redacción del manuscrito. 
de la enfermedad. Hacia 1990, la desorganización de los programas de control, la disminución de sus recursos económicos, el advenimiento de la pandemia de $\mathrm{VIH} /$ sida y la aparición de cepas resistentes al tratamiento contribuyeron a que la tuberculosis volviera a ser considerada como un problema mundial de salud pública. En 1993, la Organización Mundial de la Salud (OMS) declaró la emergencia mundial por la pandemia de tuberculosis, pues para ese año la tuberculosis ya era la primera causa de muerte debida a un agente infeccioso único (1).

Hoy en día, un tercio de la humanidad está infectada por el bacilo que causa la tuberculosis y para el 2011 había en todo el mundo alrededor de 8,7 millones de casos nuevos. Por otra parte, hay aproximadamente 347 millones de personas con diabetes y se estima que en los países con alta carga de tuberculosis, $15 \%$ de los enfermos tienen diabetes (3-5).

En el año 2011, Colombia reportó 12.701 casos para una incidencia de 25 casos por 100.000 habitantes, en tanto que la prevalencia de diabetes de tipo II está entre 4 y $8 \%$. En ese mismo año, se reportaron en Bogotá cerca de 940 casos de tuberculosis $(6,7)$ (Moreno E, Vera N. Colombia tuberculosis situation. International Congress, VI Meeting of the SLAMTB, 2012, Bucaramanga. Med UIS. 2012;(Supl.):19-20). Un informe de la Red Nacional de Laboratorios del Instituto Nacional de Salud sobre pruebas de sensibilidad realizadas entre los años 2009 y 2011 reportó los resultados de 104 aislamientos recolectados de 100 individuos que refirieron ser trabajadores al cuidado de la salud. El riesgo del personal de salud de adquirir la infección y desarrollar la enfermedad es notablemente mayor que el de la población general; en áreas con alta incidencia el riesgo puede aumentar hasta 40 veces. La diabetes mellitus es un factor de riesgo importante para la tuberculosis que, además, modifica su presentación clínica y su manejo (8-10) (Llerena $\mathrm{C}$, Vanegas A. Description of cases of tuberculosis in health workers area evaluated by the National Laboratory Network. International Congress, VI Meeting of the SLAMTB, 2012, Bucaramanga. Med UIS. 2012;(Supl.):55-6).

La tuberculosis es la enfermedad granulomatosa que con mayor frecuencia afecta la laringe y comúnmente se presenta como complicación de la tuberculosis pulmonar. La forma de presentación clínica de la tuberculosis laríngea cambió después de la introducción de la terapia antituberculosa, por lo que con frecuencia se confunde con el carcinoma laríngeo y otras patologías laríngeas. La tuberculosis laríngea primaria es rara y la ruta de infección es la invasión directa de la laringe por bacilos inhalados en gotitas contaminadas (11-14).

\section{Presentación del caso}

Se presenta el caso de un hombre mestizo de 56 años de edad, médico otorrinolaringólogo, que consultó por una disfonía progresiva de seis meses de evolución que aumentaba durante el día. En otra institución se había hecho el diagnóstico nasolaringoscópico de hemorragia en cuerda vocal izquierda y engrosamiento de pliegue vocal ipsilateral de posible etiología neoplásica.

El paciente presentaba antecedentes de diabetes mellitus de tipo Il con manejo adecuado, rinitis alérgica y enfermedad por reflujo gastroesofágico; refería fumar e ingerir alcohol tan solo ocasionalmente.

Al ingreso presentaba buenas condiciones generales, estaba hidratado y afebril; su presión arterial era de 140/80 mm Hg, la frecuencia cardiaca de 72 latidos por minuto, la frecuencia respiratoria de 18 respiraciones por minuto, su peso era de 73 $\mathrm{kg}$, su talla de $170 \mathrm{~cm}$, su superficie corporal de $1,86 \mathrm{~m}^{2}$ y su IMC de 25,26 (sobrepeso). Presentaba disfonía, y el resto del examen físico era normal. Se realizó un nuevo estudio nasoralingoscópico y se encontró "lesión epitelial con aparente componente subepitelial en tercios medio y posterior de pliegue vocal izquierdo" (figura 1).

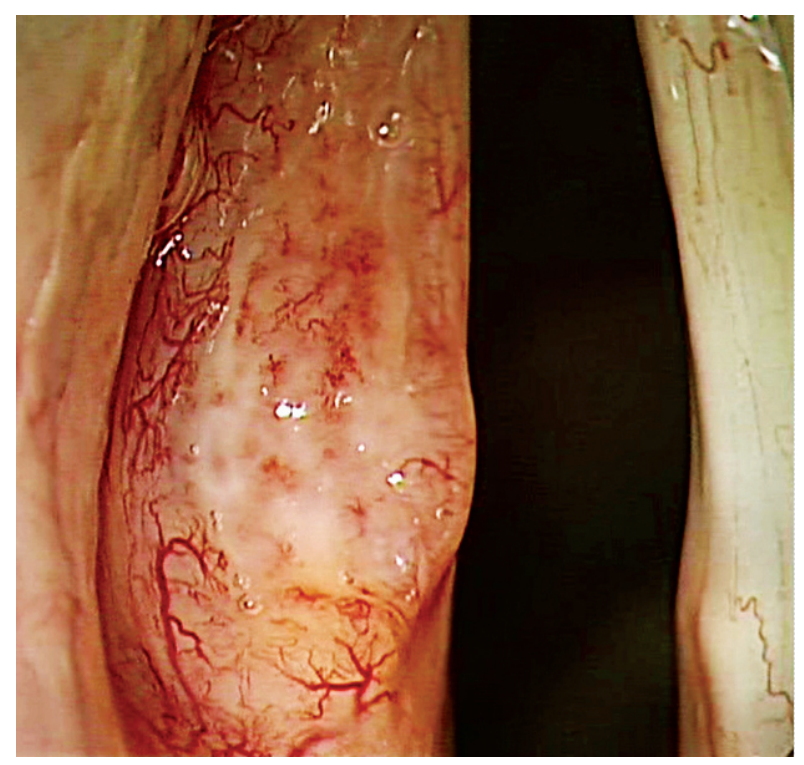

Figura 1. Lesión epitelial con aparente componente subepitelial en los tercios medio y posterior del pliegue vocal izquierdo. 
Se ordenó cuadro hemático, medición de glucemia, creatinina sérica, radiografía y tomografía de tórax con resultados dentro de los límites normales; las transaminasas séricas presentaban una ligera elevación y el trazado electrocardiográfico reveló signos que sugerían un bloqueo de rama derecha. No se determinó el estado inmune del paciente en lo tocante a tuberculosis (PPD - Interferon-Gamma Release Assays) ni a la infección por VIH. Con base en la historia clínica y los hallazgos descritos, se practicó microcirugía por laringoscopia, en la cual se tomaron muestras para la biopsia de la lesión descrita.

El espécimen quirúrgico se envió a patología y en la evaluación del tejido con la coloración de hematoxilina y eosina se reportó mucosa y tejido fibroconjuntivo comprometidos por una reacción granulomatosa, con granulomas de centro necrótico y células gigantes de tipo Langhans (figura 2); en la coloración de Ziehl-Neelsen se observaron bacilos ácido-alcohol resistentes (BAAR) (figura 3), y en las coloraciones de PAS y de Gomory no se encontraron estructuras micóticas.

Ante estos hallazgos se programó una nueva laringoscopia y se tomaron muestras para cultivo de micobacterias y para los estudios moleculares pertinentes.

En el cultivo en medio sólido de LöwensteinJensen se evidenció crecimiento a los 18 días de incubación con presencia de colonias rugosas de color marfil de aspecto cerebroide, las que examinadas con la coloración de ZN mostraron BAAR. El análisis de la muestra por PCR en busca de amplificación del fragmento IS6110 (específico del complejo $M$. tuberculosis) fue positivo. Se practicaron estudios de sensibilidad, y se demostró sensibilidad a todos los fármacos antituberculosos de primera línea. Se inició el manejo empleando la estrategia DOTS (Directly Observed Treatment, Short-course) con rifampicina, isoniacida, etambutol y pirazinamida por dos meses y rifampicina e isoniacida por cuatro meses, con lo cual se logró la curación de la enfermedad.

\section{Discusión}

La forma de presentación más común de la tuberculosis de cabeza y cuello es la de los ganglios linfáticos cervicales (90\%) seguida por la afectación laríngea (2 a $6 \%$ ). El compromiso nasal, nasosinusal, ótico, ocular, tiroideo, de garganta y de lengua es mucho menos frecuente (15-19).

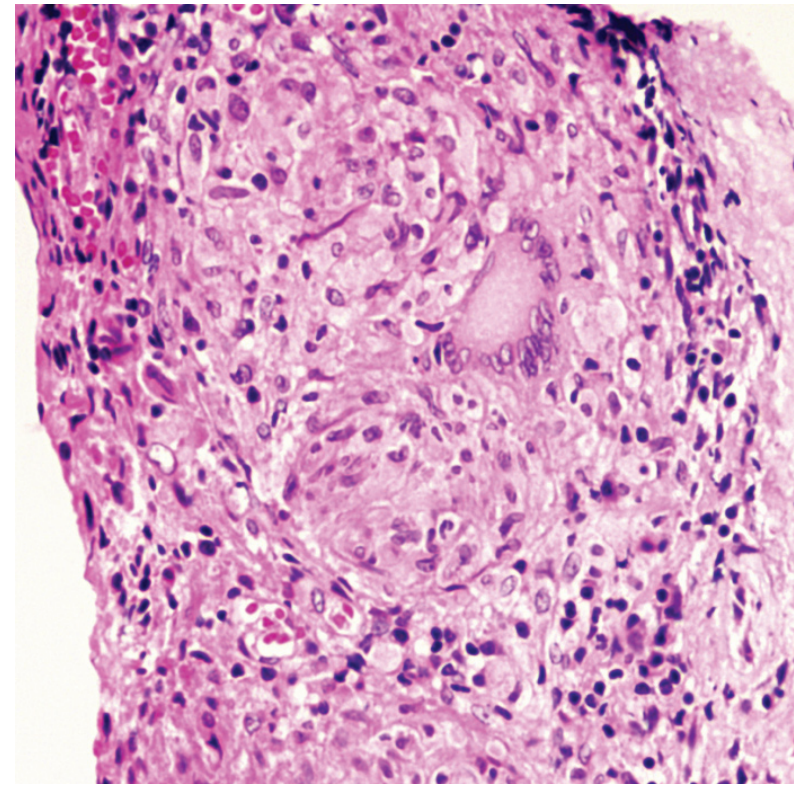

Figura 2. Tejido fibroconjuntivo correspondiente a la submucosa laríngea comprometido por una reacción granulomatosa con células gigantes de tipo Langhans. Hematoxina y eosina, 40X.



Figura 3. La flecha señala un bacilo ácido-alcohol resistente. Ziehl-Neelsen, 100X.

En la era anterior a la quimioterapia, la tuberculosis laríngea se asociaba hasta en $48 \%$ con los casos de tuberculosis pulmonar. Generalmente afectaba a jóvenes en la segunda o tercera década de la vida con tuberculosis cavitaria y frecuentemente causaba una lesión ulcerativa en la región aritenoide debido a la acumulación de esputo con bacilos viables en esta zona favorecida por la posición postrada de 
los pacientes. Después de la implementación de una terapia antituberculosa eficaz y de la mejora en las condiciones económicas y sociales de la población, entre otros factores, la presentación laríngea se hizo bastante rara. Actualmente se ve afectación de cualquier parte de la laringe y ya no hay una relación inequívoca con la tuberculosis pulmonar (15,20-22).

Hoy por hoy la tuberculosis laríngea afecta a individuos de 50 a 60 años y la relación hombre a mujer es de 1,9:1; los síntomas predominantes son disfonía (80-100 \% de los casos) y odinofagia (50-60\%), la tos varía su presentación (7-44\%), la sensación de cuerpo extraño en la garganta y la otalgia son comunes también, pero otros síntomas como la disfagia, la disnea, el estridor, la pérdida de peso, la sudoración nocturna y la hemoptisis son menos frecuentes $(13,15,19-21,23-25)$.

La laringe puede infectarse por diversas vías: a) por los bacilos expulsados en el esputo en los casos de tuberculosis pulmonar; b) por diseminación hematógena durante la micobacteriemia que suele presentarse en la infección primaria, o c) por la siembra directa de bacilos inhalados $(14,26)$.

En una serie de 60 casos de tuberculosis laríngea se encontró que las estructuras anatómicas más frecuentemente afectadas fueron, en su orden, la cuerda vocal verdadera, la cuerda vocal falsa, la epiglotis, la región aritenoide y, por último, la comisura posterior. Se asociaron a tuberculosis pulmonar activa 46,7 \% de los casos; a historia de enfermedad pulmonar o mínimas alteraciones en la radiografía de tórax, 33,3\%, y en $20 \%$ de ellos no hubo evidencia de afectación pulmonar alguna. En cinco pacientes de este estudio sólo hubo evidencia clínica de la enfermedad, sin hallazgos histológicos específicos, y las lesiones laríngeas desaparecieron después del tratamiento antituberculoso. Macroscópicamente, la enfermedad se puede presentar como una lesión granulomatosa, ulcerativa, polipoide, destructiva, o simplemente como edema y congestión local $(18,20,23,26)$. Además, se informó una relación entre las características de la lesión laríngea y la presencia de enfermedad pulmonar; así, los pacientes con tuberculosis pulmonar activa presentaron más frecuentemente lesiones laríngeas múltiples, granulomatosas o ulcerativas, mientras que los pacientes sin afectación pulmonar desarrollaron lesiones únicas, polipoides o de características inespecíficas $(24,27)$.
Las diversas formas de presentación dan pie a un amplio abanico de diagnósticos diferenciales, entre ellos, laringitis crónica, carcinoma laríngeo, enfermedad por arañazo de gato, sífilis, histoplasmosis, criptococosis, leishmaniasis, sarcoidosis, granulomatosis de Wegener, afectación laríngea por artritis reumatoide y lupus eritematoso sistémico, amiloidosis y granuloma por teflón $(11,16,28)$. De todas maneras, la entidad con la que la mayoría de las veces se confunde es con el cáncer de laringe. No obstante, se han reportado casos de tuberculosis laríngea asociados a cáncer de laringe ya manejado quirúrgica y médicamente $(16,20,22,29,30)$.

En cuanto al abordaje diagnóstico, es fundamental una historia clínica completa que indague sobre los antecedentes y eventuales factores de riesgo. En el caso aquí analizado encontramos el antecedente de diabetes mellitus de tipo II, así como la ocupación del paciente; el primero condiciona un aumento del riesgo tanto de enfermedad activa como latente (31) al modificar la respuesta inmunitaria del huésped y el segundo evidentemente aumenta el riesgo de infección por factores de exposición (32).

Como se discutió previamente, las lesiones tuberculosas de la laringe son heteromorfas y en nuestro paciente, por ejemplo, eran muy sugestivas de neoplasia; en ausencia de otros síntomas o hallazgos paraclínicos, las ayudas diagnósticas de laboratorio y patología son fundamentales para descartar el cáncer y evidenciar el proceso subyacente. Entre las técnicas de laboratorio disponibles para el diagnóstico de esta enfermedad están el análisis histológico, que detecta hasta 88 $\%$ de los casos, la tinción de Ziehl-Neelsen sobre el tejido o el esputo, el cultivo de secreciones pertinentes y la búsqueda de ADN micobacteriano mediante la técnica de reacción en cadena de la polimerasa (PCR), la cual es muy útil en el diagnóstico rápido de esta entidad y de todas las formas de tuberculosis extrapulmonar y pulmonar paucibacilar $(24,33,34)$.

La asociación de la diabetes con la tuberculosis, así como el mayor riesgo de infección o enfermedad entre los trabajadores de atención en salud, son dos situaciones siempre reconocidas y ambas de suma importancia a la hora de formular programas de control de la enfermedad. Las medidas de bioseguridad adecuadas en el contacto entre paciente y profesional de la salud son fundamentales para la protección de ambos. Un diagnóstico 
oportuno de la tuberculosis modifica el pronóstico del paciente y es el principal pilar del control de la transmisión, para lo cual es fundamental la realización de pruebas de diagnóstico rápidas y sensibles (35-38).

Un estudio realizado con trabajadores de la salud en un hospital universitario de Bogotá encontró la magnitud del problema, pues el $63 \%$ de la población encuestada creía haber estado expuesta a la tuberculosis, pero tan solo $22 \%$ manifestó usar la mascarilla N95 durante sus labores y uno de cada tres trabajadores aseguró no tener siquiera acceso a tales mascarillas. La prevalencia estimada con base en la PPD positiva fue de $23,7 \%$ (39).

La OMS recomienda la tamización para la tuberculosis en pacientes diabéticos sólo en áreas donde la prevalencia estimada de tuberculosis sea mayor de 100 casos por 100.000 habitantes (40); así pues, el médico debe estar presto a evaluar la presencia de síntomas sugestivos de tuberculosis como tos, fiebre, sudoración nocturna, pérdida de peso o dolor torácico en los pacientes diabéticos y utilizar la baciloscopia y la radiografía de tórax para la detección de la tuberculosis (41).

Todos los hospitales deberían contar con programas de vigilancia epidemiológica de la tuberculosis entre sus trabajadores y concentrar la información, lo que permitiría la realización de informes y la formulación de políticas de relevancia nacional e internacional (42).

\section{Conflicto de intereses}

Los autores declaramos no tener ningún tipo de conflicto de intereses.

\section{Financiación}

La realización del presente trabajo no requirió financiación alguna.

\section{Referencias}

1. Lienhardt C, Glaziou P, Uplekar M, Lönnroth K, Getahun H, Raviglione M. Global tuberculosis control: Lessons learnt and future prospects. Nat Rev Microbiol. 2012;10:407-16. http://dx.doi.org/10.1038/nrmicro2797

2. Kapur V, Whittam T, Musser J. Is Mycobacterium tuberculosis 15,000 years old? J Infect Dis. 1994;170:1348-9. http://dx.doi.org/10.1093/infdis/170.5.1348

3. World Health Organization. Global tuberculosis control: WHO report 2012. Geneva. Fecha de consulta: 19 de junio de 2013. Disponible en: http://apps.who.int/iris/bitstream/10 665/75938/1/9789241564502_eng.pdf.

4. World Health Organization. Diabetes. Fact sheet No. 312. Geneva: WHO; 2011. Fecha de consulta: 19 de junio de 2013. Disponible en: http://www.who.int/mediacentre/ factsheets/fs312/en/.

5. Lonnroth K, Castro KG, Chakaya JM, Chauhan LS, Floyd $\mathbf{K}$, Glaziou $\mathbf{P}$, et al. Tuberculosis control and elimination 2010-50: Cure, care, and social development. Lancet. 2010;375:1814-29. http://dx.doi.org/10.1016/S0140-6736 (10)60483-7

6. Aschner P. Epidemiología de la diabetes en Colombia. Av Diabetol. 2010;26:95-100.

7. Secretaría Distrital de Salud. Boletín Epidemiológico Distrital de Tuberculosis: Semana Epidemiológica 1 a la 52 de 2011. Bogotá, D.C. Fecha de consulta: 19 de junio de 2013. Disponible en: http://www.saludcapital.gov.co/sitios/ VigilanciaSaludPublica/Todo\%20ASIS/Boletin\%20TBC\%20 25\%20de\%20mayo.pdf.

8. Menzies D, Fanning A, Yuan L, Fitzgerald M. Tuberculosis among health care workers. N Engl J Med. 1995;332:92-8. http://dx.doi.org/10.3201/eid1703.100947

9. Sanghavi D, Gilman R, Lescano-Guevara A, Checkley W, Cabrera L, Cárdenas V. Hyperendemic pulmonary tuberculosis in a Peruvian shantytown. Am J Epidemiol. 1998;148:384-9. http://dx.doi.org/10.1371/journal.pmed. 0030494

10. Dooley KE, Chaisson RE. Tuberculosis and diabetes mellitus: Convergence of two epidemics. Lancet Infect Dis. 2009:9:737-46. http://dx.doi.org/10.1016/S1473-3099 (09) $70282-8$

11. Loehrl T, Smith T. Inflammatory and granulomatous lesions of the larynx and pharynx. Am J Med. 2001;111:113-7. http:// dx.doi.org/10.1016/S0002-9343(01)00856-7

12. Kettani N, Hassani M, Chakir N, Jiddane M. Primary laryngeal tuberculosis mimicking laryngeal carcinoma: CT scan features. Indian J Radiol Imaging. 2010;20:11-2. http:// dx.doi.org/10.4103/0971-3026.59745

13. Shin J, Nam S, Yoo S, Kim S. Changing trends in clinical manifestations of laryngeal tuberculosis. Laryngoscope. 2000;110:1950-3. http://dx.doi.org/10.1097/00005537200011000-00034

14. Kiakojuri K, Hasanjani M. Laryngeal tuberculosis without pulmonary involvement. Caspian J Intern Med. 2012;3:397-9.

15. Nalini B, Vinayak S. Tuberculosis in ear, nose, and throat practice: Its presentation and diagnosis. Am J Otolaryngol. 2006;27:39-45. http://dx.doi.org/10.1016/j.amjoto. 2005.07.005

16. Moon W, Han M, Chang K, Im J, Kim H, Sung K, Lee H. $\mathrm{CT}$ and MR imaging of head and neck tuberculosis. Radiographics. 1997;17:391-402.

17. Moon W, Han M, Chang K, Im J, Kim H, Sung K, et al. Laryngeal tuberculosis: CT findings. AJR Am J Roentgenol. 1996;166:445-9. http://dx.doi.org/10.2214/ajr. 166.2 .8553964

18. Hunter A, Millar J, Wightman A, Horne N. The changing pattern of laryngeal tuberculosis. J Laryngol Otol. 1981; 95:393-8

19. Lerra S, Nazir T, Mir Qadri S, Kirmani M. Primary nasal tuberculosis, a rare clinical entity. Ear Nose Throat J. $2011 ; 90: 12-4$.

20. Smulders Y, De Bondt BJ, Lacko M, Hodge J, Kross K. Laryngeal tuberculosis presenting as a supraglottic 
carcinoma: A case report and review of the literature. J Med Case Rep. 2009;3:9288. http://dx.doi.org/10.1186/17521947- 3-9288

21. Ullah N, Wallis S, Siddiqui N. Laryngeal tuberculosis: A diagnosis not to be missed. BMJ Case Reports. 2009;2009. http://dx.doi.org/10.1136/bcr.11.2008.1228

22. Plaza Mayor G, Pérez C, Sierra C, Rabosa García E, Fortún $\mathrm{J}$, Fogué L, et al. Laryngeal tuberculosis and laryngeal cancer. An Otorrinolaringol Ibero Am. 1998;25:387-97.

23. Harney M, Hone S, Timon C, Donnelly M. Laryngeal tuberculosis: An important diagnosis. J Laryngol Otol. 2000;114:878-80.

24. Lim J, Kim K, Choi E, Kim Y, Kim H, Choi H. Current clinical propensity of laryngeal tuberculosis: Review of 60 cases. Eur Arch Otorhinolaryngol. 2006;263:838-42. http:// dx.doi.org/10.1007/s00405-006-0063-5

25. Fernandes L, Mesqnita A. Stridor presentation in laryngeal tuberculosis. Indian J Tuberc. 1997;44:93-4.

26. Yencha M, Linfesty R, Blackmon A. Laryngeal tuberculosis. Am J Otolaryngol. 2000;21:122-6.

27. Nishiike S, Irifune M, Doi K, Sawada T Kubo T. Laryngeal tuberculosis: A report of 15 cases. Ann Otol Rhinol Laryngol. 2002;111:916-8.

28. Aspestrand F, Kolbenstvedt A, Boysen M. CT findings in benign expansions of the larynx. J Comput Assist Tomogr. 1989;13:222-5.

29. Nishiike S, Nagai M, Nakagawa A, Konishi M, Sakata Y, Aihara $\mathrm{T}$, et al. Laryngeal tuberculosis following laryngeal carcinoma. J Laryngol Otol. 2006; 120:151-3. http://dx.doi. org/10.1017/S0022215105005955

30. Cardona AF, Combariza JF, Aponte D, Pacheco J, Ospina EG, Ludovic RH, et al. Tuberculosis laríngea: neoplasia aparente. Revista Colombiana de Cancerología. 2002;6:31-9.

31. Bermejo M, Gil S, Velasco M, Prado A, Garcia C, Guijarro M. Tuberculin test in diabetic patients in a health center. Aten Primaria. 1995;16:154-7.

32. Zwerling A, van den Hof S, Scholten J, Cobelens F, Menzies D, Pai M. Interferon-gamma release assays for tuberculosis screening of healthcare workers: A systematic review. Thorax. 2012;67:62-70. http://dx.doi.org/10.1136/thx. 2010.143180
33. Chegou NN, Hoek KG, Kriel M, Warren RM, Victor TC, Walzl G. Tuberculosis assays: Past, present and future. Exp Rev Anti Infect Ther. 2011;9:457-69. http://dx.doi. org/10.1586/eri.11.23

34. Al Serhani A. Mycobacterial infection of the head and neck: Presentation and diagnosis. Laryngoscope. 2001;111:2012-6. http://dx.doi.org/10.1097/00005537-200111000-00027

35. Sterling T, Haas D. Transmission of Mycobacterium tuberculosis from Health Care Workers. N Engl J Med. 2006;355;2. http://dx.doi.org/10.1056/NEJMp068015

36. Magee MJ, Bloss E, Shin S, Contreras C, Arbanil H, Calderon $\mathrm{T}$, et al. Clinical characteristics, drug resistance, and treatment outcomes among tuberculosis patients with diabetes in Peru. Int J Infect Dis. 2013;17:e404-12. http:// dx.doi.org/10.1016/j.ijid.2012.12.029

37. Jensen PA, Lambert LA, lademarco MF, Ridzon R; CDC. Guidelines for preventing the transmission of Mycobacterium tuberculosis in health-care settings. MMWR Recomm Rep. 2005;54:1-141.

38. Dye C, Glaziou P, Floyd K, Raviglione M. Prospects for tuberculosis elimination. Annu Rev Public Health. 2013;34:271-86.http://dx.doi.org/10.1146/annurev-publhealth$031912-114431$

39. Mora ER, Pulido J, Rojas JC, Hidalgo P, Ruiz A. Conversión de tuberculina en trabajadores HUSI. Revista Colombiana de Neumología. 2011;23 (Supl.1):47-8.

40. Marais BJ, Lönnroth K, Lawn SD, Migliori GB, Mwaba P, Glaziou P, et al. Tuberculosis comorbidity with communicable and non-communicable diseases: Integrating health services and control efforts. Lancet Infect Dis. 2013;13:436-48. http:// dx.doi.org/10.1016/S1473-3099(13)70015-X

41. Lin Y, Li L, Mi F, Du J, Dong Y, Li Z, et al. Screening patients with diabetes mellitus for tuberculosis in China. Trop Med Int Health. 2012;17:1302-8. http://dx.doi.org/10.1111/j.13653156.2012.03069.x

42. Ostrosky-Zeichner L, Rangel-Frausto MS, García-Romero E, Vázquez A, Ibarra MJ, Ponce de León-Rosales S. Tuberculosis en los trabajadores de la salud: importancia de los programas de vigilancia y control. Salud Pública de México. 2000;42:48-52. http://dx.doi.org/10.1590/S003636342000000100009 